\title{
Thermodynamic Interpretation of Pressure-Induced Spin Transitions in Thiosemicarbazonates of Iron(III)
}

\author{
A. KRUPSKA* \\ Institute of Molecular Physics, PAS, M. Smoluchowskiego 17, 60-179 Poznań, Poland \\ (Received October 1, 2012; revised version April 24, 2013; in final form May 16, 2013)
}

The thermodynamic interpretation of the spin transition in iron(II) complexes taking into consideration cooperative domains is given in the literature. We use a similar interpretation for thiosemicarbazonates of aromatic aldehydes of iron(III) compounds. We extend the spin transition interpretation to pressure-induced spin transitions. The relationship between a domain size $n$ and the interaction between a high spin and a low spin states $\Gamma$ given by Gütlich et al. allows us to deduce a modified equation of the spin transition pressure $p_{\mathrm{c}}$ (at the temperature $T=T_{\mathrm{c}}$ ). On the basis of this equation, the content of the fraction of the high spin state $\gamma$ and of the fraction of the low-spin state, $1-\gamma$, as a function of pressure can be theoretically estimated. The derived equation enables us to predict the width of hysteresis which refers to the high spin and low spin states formed under the high pressure as a result of the spin transition.

DOI: $10.12693 /$ APhysPolA.124.626

PACS: 05.70.-a, 82.60.-s

\section{Introduction}

The heat capacity measurements performed in two isomorphic complexes $\left(\mathrm{Al}_{0.33}\left[\mathrm{Fe}(\mathrm{Th}-5 \mathrm{Cl}-\mathrm{Sa})_{2}\right]\right.$ and $\mathrm{H}\left[\mathrm{Fe}(\mathrm{Th}-5 \mathrm{Cl}-\mathrm{Sa})_{2}\right]$ provide approximately the same value for the transition entropy $\Delta S=17 \mathrm{~J} /(\mathrm{mol} \mathrm{K})$ within the experimental error [1]. One can suppose that this value is correct for the whole family of $\mathrm{M}\left[\mathrm{Fe}(\mathrm{Th}-5 \mathrm{Cl}-\mathrm{Sa})_{2}\right]$ compounds, in particular for $\mathrm{M}=2 \mathrm{Me}-5 \mathrm{Et}-\mathrm{PyH}$. From the Clausius-Clapeyron relation, $\mathrm{d} p_{\mathrm{c}} / \mathrm{d} T_{\mathrm{c}}=\Delta S / \Delta V$ (where $\mathrm{d} p_{\mathrm{c}}$ is a small variation of the spin-transition pressure, $\mathrm{d} T_{\mathrm{c}}$ is a small variation of the spin-transition temperature, $\Delta S$ - entropy change, $\Delta V$ - volume change) applied to the transition from the high-spin (HS) state to the low pressurelow spin state LS- 1 , the volume change is estimated as $\Delta V=2.2 \mathrm{~cm}^{3} / \mathrm{mol}$. However, for the transition from the LS-1 state to the high pressure-low spin state LS-2 $\mathrm{d} p_{\mathrm{c}} / \mathrm{d} T_{\mathrm{c}} \approx 0$, so the change of the transition entropy (as follows from Clausius-Clapeyron relation) can be neglected. In order to find the volume and entropy changes in the transition from the HS state to the LS-2 state we have applied the additive relations: a slope on temperature equal to $2.6 \mathrm{MPa} / \mathrm{K}$ in the neighborhood of the triple point for these quantities. It follows that the entropy of the transition from the HS to the LS-2, HS $\leftrightarrow$ LS-2, is equal to $7.7 \mathrm{~J} /(\mathrm{mol} \mathrm{K}$ ) (as obtained from the slope coefficient $\left.\mathrm{d} p_{\mathrm{c}} / \mathrm{d} T_{\mathrm{c}}\right)[2,3]$.

The thermodynamic interpretation of the spin transition in iron(II) complexes taking into consideration cooperative domains is given in some papers $[1,4,5]$. A phenomenological model of the spin transition in iron(II)

*e-mail: krupska@ifmpan.poznan.pl is presented in [6]. We have used a similar interpretation for thiosemicarbazonates of aromatic aldehydes of iron(III) compounds, where formation of LS domain in $\mathrm{Na}\left[\mathrm{Fe}(\mathrm{Th}-\mathrm{Sa})_{2}\right.$ ] was proved [7]. The EPR linewidth analysis has shown that LS complexes formed are not statistically distributed among HS ones but aggregate in domains [7].

\section{Thermodynamic model of pressure spin-transition}

The HS fraction $\gamma$ is expressed as

$$
\gamma=\frac{I_{\mathrm{HS}}}{I_{\mathrm{LS}}+I_{\mathrm{HS}}} \text {. }
$$

The Gibbs free energy $G$ depends on mixing entropy $S_{\text {mix }}$ as well as on the interaction parameter $\Gamma$ between the HS and LS phases and can be written for 1 mole of the iron complexes in the form

$$
G(p, T, \gamma)=\gamma G_{\mathrm{H}}+(1-\gamma) G_{\mathrm{L}}-T S_{\text {mix }}+\gamma(1-\gamma) \Gamma,
$$

where

$$
S_{\text {mix }}=-\frac{R}{n}[\gamma \ln \gamma+(1-\gamma) \ln (1-\gamma)] .
$$

Here $R$ is the gas constant, $n$ is the domain size, i.e. the number of close ions of similar spin (low or high) simultaneously changing their spin state through cooperative spin-phonon interaction, $G_{\mathrm{H}}$ and $G_{\mathrm{L}}$ are the Gibbs energies in the HS and LS states, respectively.

At equilibrium the Gibbs function (1) must have a minimum i.e.

$$
\left(\frac{\partial G}{\partial \gamma}\right)_{T, p}=0
$$

This condition yields the equation for $\gamma=\gamma(p, T)$ :

$$
\Delta G+\frac{R T}{n} \ln \frac{\gamma}{1-\gamma}+(1-2 \gamma) \Gamma=0 .
$$

In an isobaric process, the change of the Gibbs energy 
$\Delta G$ for the pressure-induced HS $\leftrightarrow$ LS transition is expressed by changes of the Helmholtz free energy $\Delta F$ and volume $\Delta V$ :

$$
\Delta G=\Delta F+p \Delta V .
$$

The spin transition pressure $p_{\mathrm{c}}$ (at constant temperature $T=T_{\mathrm{c}}$ ), by definition, corresponds to $\gamma=1 / 2$, in which case, from Eq. (5) $\Delta G=0$, so that $p_{\mathrm{c}}=-\Delta F / \Delta V$. Inserting Eq. (6) in (5) the following equation of the spin state is obtained:

$$
\left(p-p_{\mathrm{c}}\right) \Delta V+\frac{R T_{\mathrm{c}}}{n} \ln \frac{\gamma}{1-\gamma}+(1-2 \gamma) \Gamma=0 .
$$

The domain size $n$ and the parameter $\Gamma$, in principle, express the same cooperative interaction between the ion complexes in the same spin state, e.g., electron-phonon coupling or elastic interactions. The relation between $n$ and $\Gamma$ derived by Gütlich et al. [6] is given by

$$
n=1 /\left(1-\frac{\Gamma}{2 R T_{\mathrm{c}}}\right) \text {. }
$$

Combining Eqs. (8) and (7) yields a modified equation of the spin state

$$
\begin{aligned}
& \left(p-p_{\mathrm{c}}\right) \Delta V+\frac{R T_{\mathrm{c}}}{n}\left[\ln \frac{\gamma}{1-\gamma}+2(n-1)(1-2 \gamma)\right] \\
& \quad=0,
\end{aligned}
$$

similar to the Van der Waals equation for a real gas. Depending on the $n$ value, the $\gamma(p)$ curve constant $T$, can be gradual, steep or show a hysteresis (Fig. 1) critical $n$ value, $n_{\mathrm{c}}$, corresponding to the steep curve. $n_{\mathrm{c}}$ can be calculated from the curve $p(\gamma)$. By taking the derivative of Eq. (9) with respect to $\gamma$ at $\gamma_{c}=1 / 2, n_{c}=2$ is found. For $n<2$ the transition is gradual and for $n>2$ the transition possesses a hysteresis.

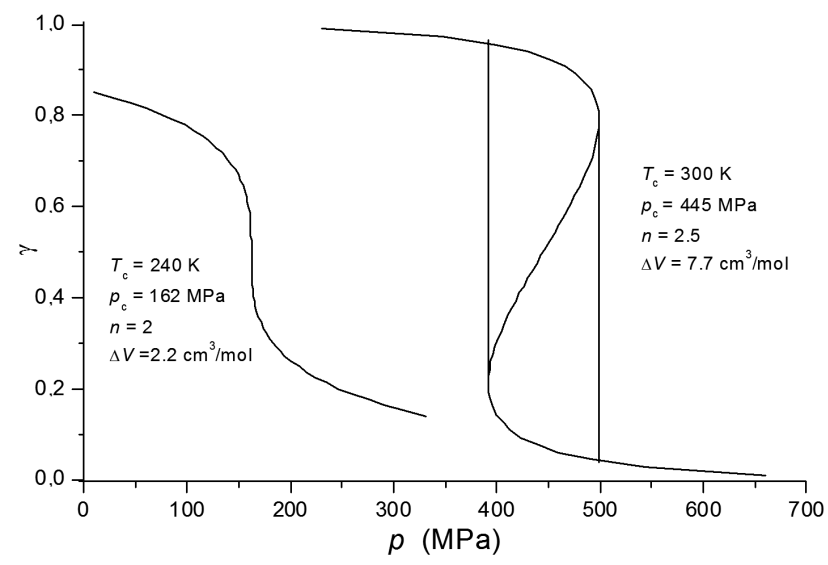

Fig. 1. The content of the fraction of HS phase $\gamma$ as a function of pressure calculated from Eq. (9). The parameters used in the calculation are given in the figure.

In an isothermal process

$$
\Delta G=\Delta H-T \Delta S,
$$

where $\Delta H$ and $\Delta S$ are the enthalpy and entropy changes, respectively. Inserting Eq. (10) in (5) at the transition temperature $\Delta G=0$ and $T_{\mathrm{c}}=\Delta H / \Delta S$, so, the equation of spin state takes the form:

$$
\left(T-T_{\mathrm{c}}\right) \Delta S-\frac{R T}{n} \ln \frac{\gamma}{1-\gamma}-(1-2 \gamma) \Gamma=0 .
$$

From (9) and (11) also results that $n_{\mathrm{c}}=2$ (by taking the derivative of Eq. (11) with respect to $\gamma$ at $\gamma_{c}=1 / 2$ after substitution into Eq. (8)).

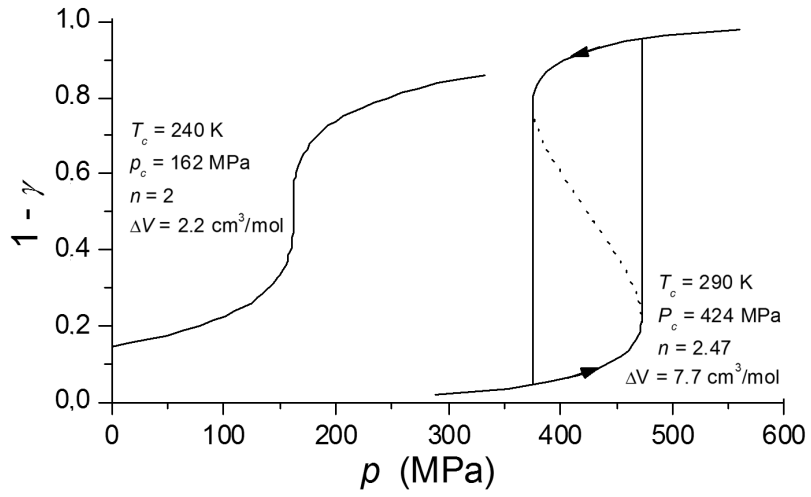

Fig. 2. The content of the fraction of LS phase, $1-\gamma$, as a function of pressure calculated from Eq. (9). The parameters used in the calculation are given in the figure.

The content of LS phase, $1-\gamma$, as a function of pressure is shown in Fig. 2. The left curve corresponds to the point $(240 \mathrm{~K}, 162 \mathrm{MPa})$ lying on the border of the HS $\leftrightarrow$ LS transition for which very narrow hysteresis (less than 0.5 MPa) appears. As follows from our calculations, the domain size $n=2$ is critical. The right curve is plotted at the point $(290 \mathrm{~K}, 424 \mathrm{MPa})$ and shows a hysteresis (dotted line in Fig. 2) of HS $\leftrightarrow$ LS transition. In order to obtain a proper hysteresis width the value of $n=2.47$ has been used in the calculation (Fig. 2).

\section{Discussion}

The thermodynamic model of the spin transitions presented above refers to thiosemicarbazonate of iron(III) under high hydrostatic pressure at a constant temperature. The existence of cooperative domains is taken into account. This thermodynamic interpretation can explain some aspects of our experimental EPR studies under high pressure performed for these types of thiosemicarbazonates of iron(III) $[2,3,7]$.

The detailed analysis of the EPR data concerns only the LS state $[2,3,7,8]$. The EPR powder spectrum of the HS state is broad because of the fine structure and dipole-dipole interactions between the magnetic neighbors, and its detailed analysis is very difficult to carry out. For this reason the HS EPR spectrum is estimated by superposition of two Lorentz lines, from which the integral intensity of the HS state $I_{\mathrm{HS}}$ is calculated. Below the critical temperature $T_{\mathrm{c}}=187 \mathrm{~K}$ the integral intensity of the LS state, $I_{\mathrm{IS}}$ can be separated from $I_{\mathrm{HS}}$ by the fitting procedure described in our previous papers $[3,8]$. 
The thermodynamic parameters used for its description relate to very limited regions of the crystal.

The very small domain sizes are obtained from the thermodynamic calculation. It means that connecting only two LS molecules into domains significantly changes the spin-phonon interaction as well as the mixing entropy. The critical domain size $n_{\mathrm{c}}=2$ is less than $n \approx \approx 3.5$ estimated by Güülich et al. [6] for the weakly coupled system $\left[\mathrm{Fe}(2 \text {-pic })_{3}\right] \mathrm{Cl}_{2} \cdot \mathrm{C}_{2} \mathrm{H}_{5} \mathrm{OH}$, and it is considerably smaller than the value of $n \approx 90$ obtained by Sorai and Seki [4] for the system with strong intermolecular coupling $\left[\mathrm{Fe}(2-\mathrm{pic})_{3}(\mathrm{NCS})_{2}\right]$. Equation (9) is derived for constant $n$ independent of pressure, therefore, it can be applied only in a narrow pressure range near $p_{\mathrm{c}}$. Thus, the shape of the $\gamma(p)$ curve cannot be properly reproduced. Nevertheless, the main characteristics of the spin transition can be described by means of this equation.

We assume that between adjacent paramagnetic centers there are weak exchange interactions. As a result, a change in spin state in one complex causes a change in the spin state in the neighboring complexes. The low-dimensional domains with $n=2$ are formed in particular. The mechanism of inhibiting the formation of domains with high $n$ can be a strain in the domain environment due to the different dimensions of the HS and LS ions. If the domains are larger, changes in the structure are also larger, as was observed for the transition $\mathrm{HS} \leftrightarrow \mathrm{LS}-2$ in $\mathrm{Na}\left[\mathrm{Fe}(\mathrm{Th}-\mathrm{Sa})_{2}\right]$ [7].

The process of domain formation can be specific to different compounds; however, the main features of its behavior under the change of temperature or pressure are preserved [9]. One can suppose that the thermodynamic interpretation presented in this paper is also relevant to all compounds of thiosemicarbazonates of iron(III), at least, as far as pressure-induced transitions are concerned.

\section{Conclusions}

A modified equation of the pressure-induced spin transition has been obtained. Based on this equation, the contents of the HS and LS phases can be evaluated as a function of hydrostatic pressure. This relation enables also to predict the existence of hysteresis and its width.

One can suppose that the thermodynamic interpretation presented above can be useful for theoretical prediction of HS $\leftrightarrow$ LS transitions with connection with domain formation, especially under application of high pressure.

\section{References}

[1] V.I. Shipilov, V.V. Zelentsov, V.M. Zhdanov, V.A. Turdakin, Zh. Eksp. Teor. Fiz. 19, 560 (1974) (in Russian).

[2] A. Krupska, M.A. Augustyniak-Jabłokow, V.Yu. Yablokov, V.V. Zelentsov, Acta Phys. Pol. A 108, 291 (2005).

[3] A. Krupska, M.A. Augustyniak-Jablokow, Yu.V. Yablokov, V.V. Zelentsov, V.A. Ulanov, J. Mrozinski, Acta Phys. Pol. A 110, 81 (2006).

[4] M. Sorai, S. Seki, J. Phys. Chem. Solids. 35, 555 (1974).

[5] A. Michalowicz, J. Moscovici, J. Charton, F. Sandid, F. Benamrane, Y. Garcia, J. Synchr. Rad. 8, 701 (2001).

[6] P. Gütlich, H. Köppen, R. Link, H.G. Steinhäuser, J. Chem. Phys. 70, 3977 (1979).

[7] V.V. Zelentsov, Yu.V. Yablokov, M. AugustyniakJablokow, A. Krupska, J. Mrozinski, V.A. Ulanov, Chem. Phys. 301, 15 (2004).

[8] Yu.V. Yablokov, V.V. Zelentsov, M.A. AugustyniakJablokow, A. Krupska, J. Mrozinski, Mater. Sci. 21, 215 (2003).

[9] Yu.V. Yablokov, V.V. Zelentsov, M.A. AugustyniakJablokow, A. Krupska, J. Mrozinski, in: Modern Problems in Condensed Matter Physics, Eds. B.Z. Malkin, Yu.N. Proshin, CJC "Novoe Znanie" (New Knowledge), Kazan 2004, p. 367. 